\title{
Giant Rectus Sheath Hematoma Causing Abdominal Compart- ment Syndrome: Review and Management
}

\author{
Ana María Minaya Bravo*, Rita Esther Medina Quintana, Fernado Mendoza Moreno, Julio Cesar Garcia Mahillo
}

Department of General and Digestive Surgery. Principe de Asturias Hospital, Alcala de Henares, Madrid. Spain

"Corresponding author: Ana María Minaya Bravo, Department of General and Digestive Surgery. Principe de Asturias Hospital, Carretera Alcalá-Meco, s/n, 28805 Alcalá de Henares, Madrid, Spain. Tel: +34918878100; E-mail: ana-minaya@hotmail.com

Citation: Minaya Bravo AM, Medina Quintana RE, Mendoza Moreno F, Garcia Mahillo JC (2017) Giant Rectus Sheath Hematoma Causing Abdominal Compartment Syndrome: Review and Management. J Surg 2017: JSUR-148. DOI: 10.29011/JSUR148.000048

Received Date: 10 June, 2017; Accepted Date: 29 June, 2017; Published Date: 06 July, 2017

\begin{abstract}
Rectus Sheath Hematoma (RSH) is a rare condition that frequently affect elderly women on anticoagulant therapy. Risk factors include trauma, surgery, cough, iatrogenic, renal or blood disorders. Most patients present a painful mass at the moment of admission, accompanied of a drop of hemoglobin level. Although the vast majority of cases are self-limiting, and managed with conservative treatment (anticoagulation reversal, fluid replacement, blood transfusion, analgesics), there is still a minority of cases that will present hemodinamically inestability and will need selective embolisation or even surgery. US and CT are useful imaging techniques, especially intravenous contrast CT that will select patients for embolisation. Management is a dilemma, and decision of when reintroducing anticoagulation can convert into a challenge. There is a lack of consensus and guidelines about how to manage this entity, and most of literature consist of series of case reports. We report a case of giant rectus sheath hematoma that caused abdominal compartment syndrome and required two embolisation and surgery. This is a peculiar case because although rectus sheath hematoma has been reported, previously, as cause of abdominal compartment syndrome, this is an uncommon presentation and few cases reported in the English literature.
\end{abstract}

\section{Introduction}

Rectus Sheath Hematoma (RSH) is an un common entity in clinical practice. It typically appears in elderly patients on anticoagulant therapy, as a result of trauma, surgery, pregnancy, paroxysmal coughing and other medical disorders. They are more frequent in women and located on infraumbilical region. It represents less than $2 \%$ of the total cases of abdominal pain collected in the Emergency Department [1-5]. RSH usually presents as a painful mass associated with a significant decrease in hemoglobin levels. Occasionally, they may raise a problem in differential diagnosis. Computed Axial Tomography (CT) and Abdominal Ultrasound (US) are useful diagnostic techniques [1-5]. Most of them are a self- limiting process that respond to non-aggressive treatment. However, some cases will need selective embolisation or surgery $[6,7]$.

We report a case of giant RSH in an 80-year-old woman that was on anticoagulant therapy. The peculiarity of this case is that RSH caused abdominal compartment syndrome and required drain and two selective embolisations. Abdominal compartment syndrome produced by RSH has been previously described in the literature, although it is a rarity.

\section{Case Report}

A 80 year old female with a medical history of arterial hypertension, rheumatic valvulopathy with mechanical mitral prosthesis and tricuspid annuloplasty, severe pulmonary hypertension and atrial fibrillation. She was on anticoagulant therapy (acenocumarol) and had been admitted two months earlier to our center due to lower leg hematomas. As a result, at the moment of admission, she was on Low-Molecular-Weight Heparin (LMWH). The patient presented with a 12-hour history of diffuse abdominal pain, nausea, abdominal distension and mass effect.

On physical examination, she was hemodynamically stable with tenderness and palpable abdominal mass on the hypogastrium. Blood tests revealed: hemoglobin level $10 \mathrm{~g} / \mathrm{dL}$, hematocrit 34\%, platelet 177.000/UL, Activated Partial Tromboplastin Time 


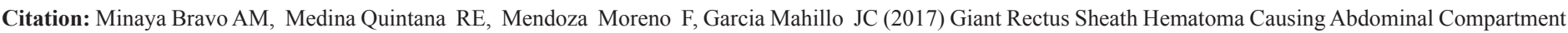
Syndrome: Review and Management. J Surg 2017: JSUR-148.

(APTT) $28.9 \mathrm{sec}$ and International Normalized Ratio (INR) 1.07. Under the suspicion of a RSH, Computed Tomography Angiography (CTA) was performed. It showed a $20 \times 25 \mathrm{~cm}$ size rectus sheath hematoma with active bleeding signs (Figure 1).
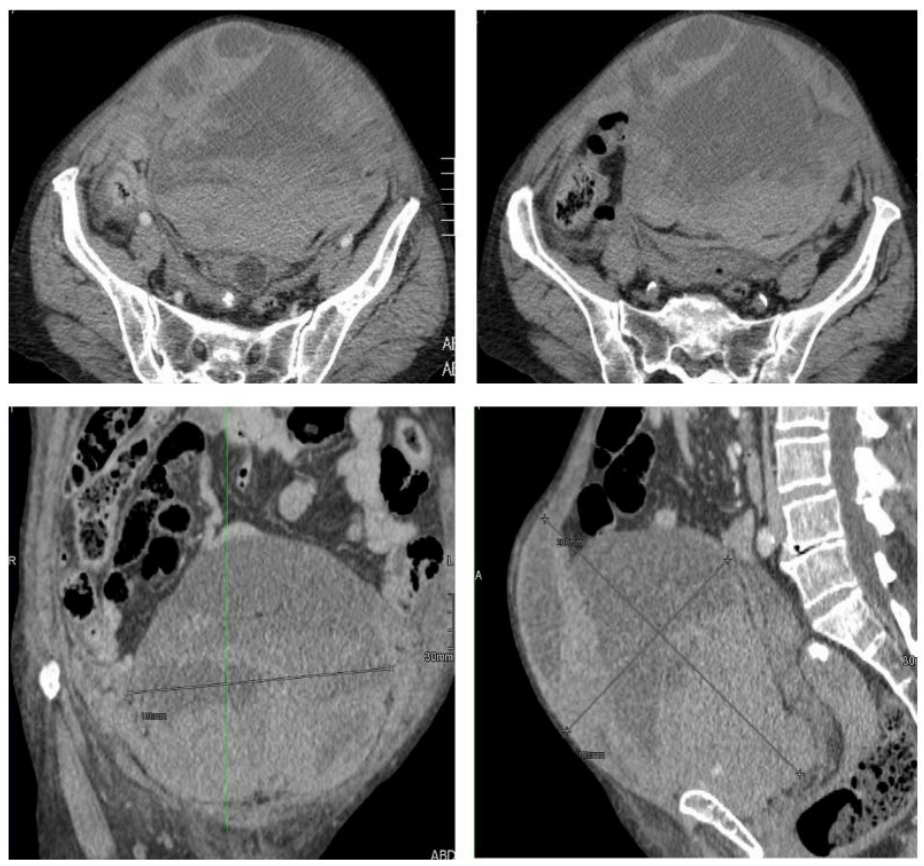

Figure 1: CTA showed a $20 \times 25 \mathrm{~cm}$ size rectus sheath hematoma with active bleeding signs.

While the patient was in the Emergency Department, her hemoglobin level plummeted from 10 to $6 \mathrm{~g} / \mathrm{dl}$ accompanied by hypotension and tachycardia. Intravenous fluid replacement, red blood cell and fresh frozen plasma transfusion were started. At that moment, the patient was moved to the Intensive Care Unit, where she was stabilized. Since there was an active bleeding, a therapeutic angiography with embolisation of the left inferior epigastric artery was carried out. In the following days, the patient developed an abdominal compartment syndrome with $23 \mathrm{~mm} \mathrm{Hg}$ of intra- abdominal pressure, and acute renal failure due to giant preperitoneal hematoma. She underwent urgent surgery. A small infraumbilical laparotomy allowed to get into Retzius space. A damaged rectus muscle was found, so epigastric vessels couldn't be identified. Clot evacuation and packing were performed. Then, 24 hours later, it was removed and no active bleeding found. In the next $72 \mathrm{~h}$ hours, the hemoglobin level dropped from 11 to $8 \mathrm{~g} / \mathrm{dl}$. A repeated CT showed active bleeding signs, and another embolization of the left inferior epigastric artery was realized (Figure 2 and 3).
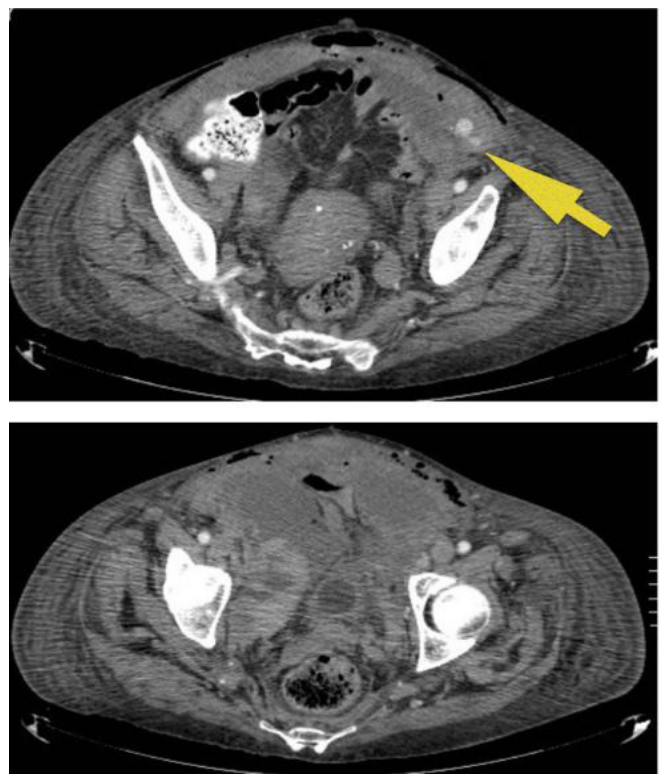

Figure 2: A repeated CT showed active bleeding signs and rest of hematoma.
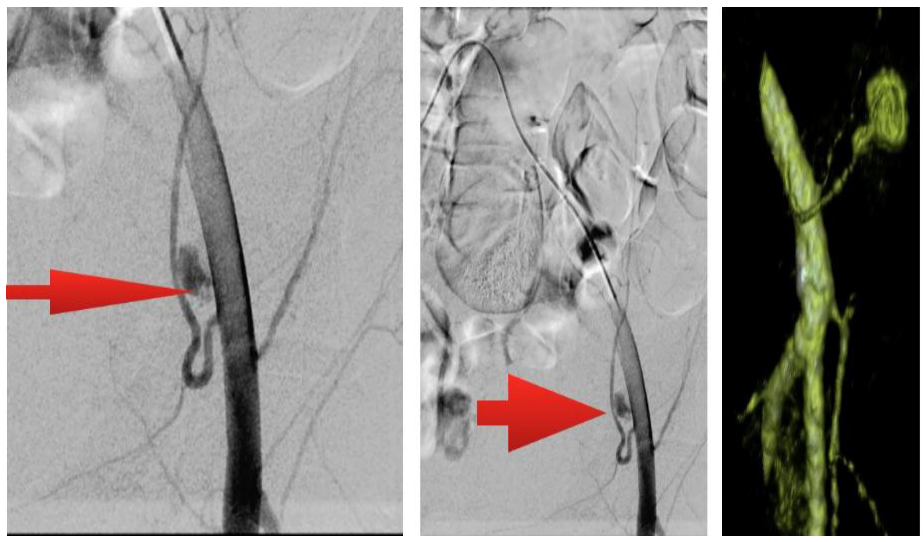

Figure 3: Embolization of the left inferior epigastric artery.

Finally, the patient stayed stable with hemoglobin level 11 $\mathrm{g} / \mathrm{dl}$, and anticoagulant therapy reintroduced. However, due to her medical conditions she suffered from heart failure and passed away.

\section{Discussion}

In recent years, the use of anticoagulant drugs in elderly, has caused an increasing number of Rectus Sheath Hematoma (RSH). RSH constitutes the most common non-neoplastic disorder occurring in Rectus Abdominis Muscles (RAM) [1]. The ancients al- 


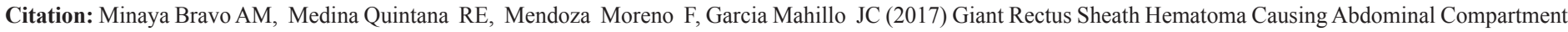
Syndrome: Review and Management. J Surg 2017: JSUR-148.

ready described this entity, and Hippocrates and Galen mentioned it in their treatises. Later, in 1857, Richardson reported the first case in the modern literature. $[2,3,4]$. In 1946, Teske analyzed a series of 100 cases of hematoma of the anterior rectus abdominis muscle [8]. According to Klinger et al. a RSH is discovered in $1.8 \%$ of patients who undergo ultrasonography for acute abdominal disorder [1]. They mostly affect women in their sixth and seventh decades of life $[2,3,6]$. On contrary, Kingler in his 23 patient serie reported a slightly predilection for men [1]. RSH is caused by a tear of rectus abdominis muscles or epigastric vessels. There are two epigastric vessels: the superior epigastric artery (terminal branch of internal thoracic artery) and inferior epigastric artery (branch of external iliac artery). Most frequently, RSH is located below the arcuate line of Douglas, where posterior surface of RAM is only covered by a weak transversalis fascia and peritoneum. (Figure 4).

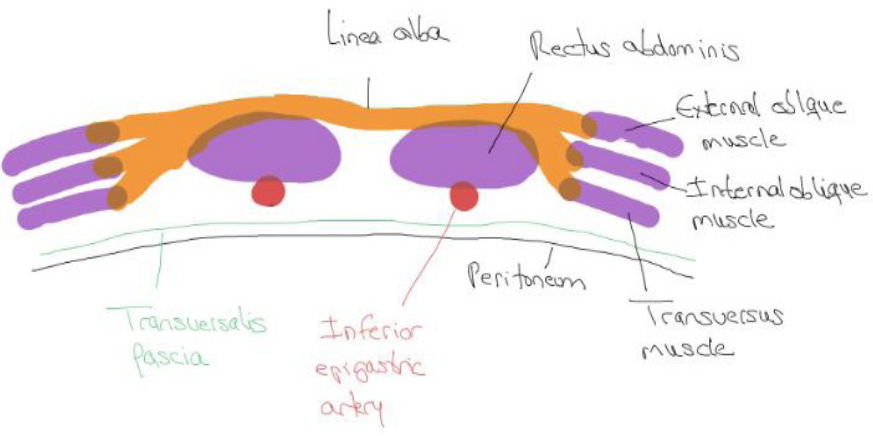

Figure 4: Below arcuate line, posterior surface of RAM is only covered by a weak transversalis fascia and peritoneum.

According to Nikolao, $70 \%$ of RHSs occur in the lower abdomen [2]. However, above arcuate line, aponeurotic expansions of external oblique, internal oblique and transversalis muscles pass anteriorly and posteriorly the RAMs, and form a thick sheath that grants contention. They appear most commonly in the right side [2-6,9] (Figure 5).

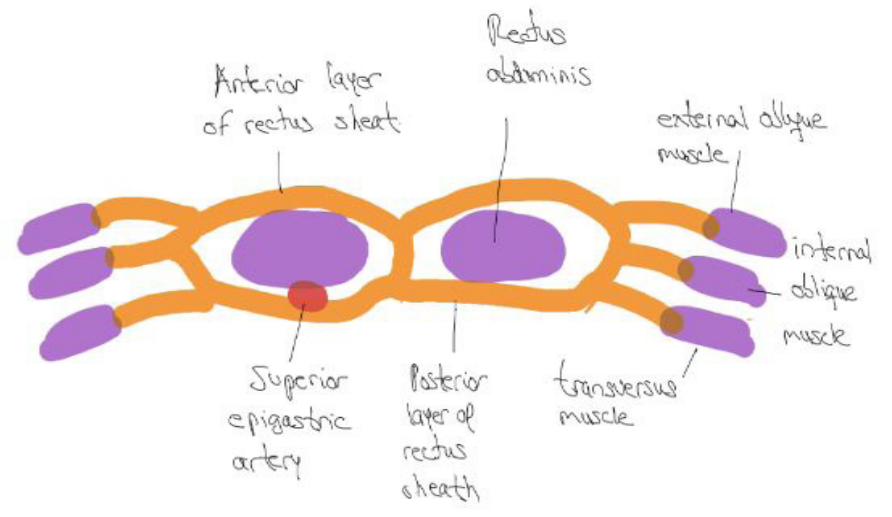

Figure 5: Above arcuate line, aponeurotic expansions of external oblique, internal oblique and transversalis muscles pass anteriorly and posteriorly the RAMs.
Several risk factors have been associated to RSH, among them, the most common is anticoagulant therapy that is also related to increasing mortality. Overall mortality rate of RSH is, approximately, 4\%. However, in patients under anticoagulant therapy, it reaches around $25 \%[1,5]$. Other causes of RSH are abdominal direct trauma, iatrogenic (procedures such as paracentesis, percutaneous drain, laparoscopic...etc.), Valsalva effort (coughing) pregnancy, elderly (weak RAMs contribute to formation of RHS) and medical conditions as hypertension, atherosclerosis, vasculitis, renal or blood disorders... Cough constitutes the most frequent precipitating factor $[1-3,5]$. They usually present as a painful mass in elderly patients on anticoagulant medications. Pain occurs in $85-97 \%$ of patients, it appears suddenly and can mimic acute intra-abdominal conditions such as appendicitis, perforated ulcer, intestinal obstruction, strangulated hernia, ectopic pregnancy...etc $[1,3,5,8,10]$. In 1926, two clinical signs were described. Fothergill's sign occurs when a palpable mass in the abdominal wall does not cross the middle line. Carnett's sign is positive when at tensing abdominal wall, tenderness increases. However, in case of intraabdominal process, the fact of lifting head and shoulders decreases the pain due to protection of abdominal wall $[3,6,11]$.

US is the first-line imagining choice, and it reaches a sensitivity of 70-90\%. However, CT is considered the gold standard with a sensitivity of $100 \%$. It allows to exclude other abdominal processes. Furthermore, it determines the size of hematoma and signs of active bleeding when realized with intravenous contrast, and identifies cases that will be candidates to selective embolisation.

In 1996, Berna proposed a classification based on CT findings:

-Type I: unilateral hematoma that does not require hospitalization, mild, and intramuscular

-Type II: moderate intramuscular hematoma that requires hospitalization, unilateral or bilateral.

-Type III: severe hematoma on anticoagulated patients that extends into peritoneum and prevesical space and requires blood transfusion [7]. The vast majority of RSHs are self-limiting and conservative treatment is successful in most cases. Non- surgical management consists of suspension of anticoagulants, blood transfusion, rest and antibiotic [6]. Reversal of anticoagulation is usually needed and consist of intravenous vitamin K; prothrombin complex concentrates or fresh frozen plasma [7].

However, we emphasize that the main data to decide the correct treatment is the hemodynamic stability of the patient. When hemodynamic instability is present, selective embolisation or surgery should be considered. In addition, enlarging hematomas should undergo embolisation $[2,3,12]$. Surgery consist of ligation of vessels or drainage of the hematoma [6]. We report a case of a giant RSH that caused abdominal compartment syndrome and, al- 


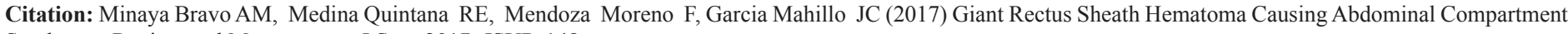
Syndrome: Review and Management. J Surg 2017: JSUR-148.

though initially selective embolisation was successful, the patient required surgery because of the high intra-abdominal pressure. A 3 $\mathrm{cm}$ infraumbilical incision was performed and middle line opened, the giant hematoma was drained but no bleeding vessel identified, although a diffuse bleeding was present and a packing performed. Two days later, packing was removed and no signs of bleeding identified. However, three days later, the hemoglobin plummeted, and a second embolisation was carried out. This is a rare case where a second embolisation was needed.

$\mathrm{RSH}$ is a rare cause of abdominal compartment syndrome, and although described in the literature, is a rare presentation. When it occurs, surgery is mandatory to reduce intra-abdominal pressure. When anticoagulation reversal has been required, a critical issue is to determine the adequate time to reintroduce anticoagulant therapy. The fact that most patients with RHS need anticoagulation for life-threading conditions (mechanical valves, auricular fibrilation...etc) makes it challenging. It is commonly accepted to continue with anticoagulant therapy once the patient is hemodynamically stable. In addition, Villa et al. recommend a repeated contrast-enhanced CT in patients under anti-coagulation [13]. In our case, once patient was hemodynamically stable, anticoagulation was initiated LMWHs require special concern because of the high number of cases of severe RSH due to injection technique. On this way, the deltoid region could be a safer alternative [2].

\section{Conclusion}

To summarize, RSH occurs mostly in anticoagulated elderly on infraumbilical location. US and CT are useful tools and allow to identify patients that will benefit of selective embolisation. Although, most cases are self-limiting, and nonaggressive treatment will be effective, some patients will present active bleeding and hemodynamically inestability; under these circumstances, selective embolisation should be performed, and finally, surgery considered. The fact that RSH happens in patients with life-threading pathologies make the management a challenge.

\section{References}

1. PJ Klingler G, Wetscher, K Glaser, J Tschmelitsch, T Schmid, et al. (1999) The use of ultrasound to differentiate rectus sheath hematoma from other acute abdominal disorders. Surg Endosc 13: 1129-1134.

2. Nikolaos S, Gourgiotis S, Karalis G (2010) Diagnostic evaluation and management of patients with rectus sheath hematoma. A retrospective study. Int J Surg 8: 290-293.

3. Hatjipetrou A, Anyfantakis D, Kastanakis M (2015) Rectus sheath hematoma: A review of the literature. Int J Surg 13: 267-271.

4. Chang WT, Knight WA, Wederhoff SG (2009) Rectus sheath hematoma, www.emedicine.comweb; July 1, 2009. site; updated.

5. Buffone A, Basile G, Costanzo M, Veroux M, Terranova L, et al. (2015) Management of patients with rectus sheath hematoma: personal experience. J Formos Med Assoc 114: 647-651.

6. Aktürk OM, Kayılıoğlu SI, Aydoğan I, Dinç T, Yildiz B, et al. (2015) Spontaneous Rectus Sheath Hematoma: An Overview of 4-Year Single Center Experience. The Indian Journal of Surgery 77: 1219-1221.

7. JD Berna, I Zuazu, M Madrigal, V Garcia-Medina, C Fernandez, et al. (2000) Conservative treatment of large rectus sheath hematoma in patients undergoing anticoagulant therapy. Abdominal Imagining 25: 230-234.

8. Teske JM (1946) Haematoma of the rectus abdominis muscle: report of case and analysis of 100 cases from literature. Am J Surg 71: 689-695.

9. Mendoza Moreno F, Diez Alonso M, Villeta Plaza R, Minaya Bravo A, Ovejero Merino E, et al. (2016) Spontaneous haematoma of the anterior rectus abdominis muscle. Cir Esp 94: 294-299.

10. Cherry WB and Mueller PS (2006) Rectus sheath hematoma. Review of 126 cases at a single institution. Medicine 85: 105-110.

11. Fothergill WE (1926) Hematoma in the abdominal wall simulating pelvic new growth. Br Med J 6: 941-942.

12. Palatucci V, Lombardi G, Lombardi L, Giglio F, Giordano F, et al (2014) Spontaneous muscle haematomas: management of 10 cases. Translational Medicine 10: 13-17.

13. M Villa, M Grande, F Ruling, D Korda (2012) Rectus sheath haematoma: are there prognostic risk factors of hemodynamic inestability motivating an early operative treatment? Eur J trauma Emerg Surgery 38: $537-541$ 